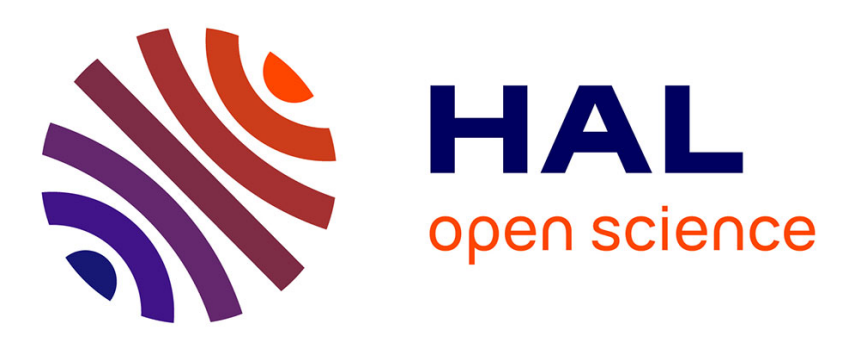

\title{
Nouveaux produits de la pharmacopée chinoise contemporaine : $R \& D$, définition et socialité en réseaux
}

Evelyne Micollier

\section{To cite this version:}

Evelyne Micollier. Nouveaux produits de la pharmacopée chinoise contemporaine: R\&D, définition et socialité en réseaux. Autrepart - Revue de sciences sociales au Sud, 2012, 63, pp.69-88. 10.3917/autr.063.0069 . halshs-01069102

\section{HAL Id: halshs-01069102 \\ https://shs.hal.science/halshs-01069102}

Submitted on 16 May 2017

HAL is a multi-disciplinary open access archive for the deposit and dissemination of scientific research documents, whether they are published or not. The documents may come from teaching and research institutions in France or abroad, or from public or private research centers.
L'archive ouverte pluridisciplinaire HAL, est destinée au dépôt et à la diffusion de documents scientifiques de niveau recherche, publiés ou non, émanant des établissements d'enseignement et de recherche français ou étrangers, des laboratoires publics ou privés. 
Micollier Évelyne, "Nouveaux produits de la pharmacopée chinoise contemporaine : R\&D, définition et socialité en réseaux ", Autrepart, 2013/1 No63, p. 69-88. DOI : 10.3917/autr.063.0069

\section{Nouveaux produits de la pharmacopée chinoise contemporaine : $R \& D$, définition et socialité en réseaux}

\section{Évelyne Micollier* ${ }^{1}$}

Les médicaments pharmaceutiques fabriqués industriellement sont aujourd'hui présents partout dans le monde, en Chine et ailleurs, loin de leurs terres d'origine, celles de la révolution industrielle. L'industrie du médicament occupe une place de choix, au sein du secteur secondaire de l'économie, qui s'est renforcée avec les nouvelles formes de mondialisation, dénommées «globalisation» pour les distinguer des formes antérieures. Ong et Collier [2005] notent qu'il n'y a pas d'accord entre les disciplines ni entre les auteurs sur une définition consensuelle de la globalisation. Trois aspects récurrents dans les multiples définitions résonnent plus que d'autres avec l'analyse développée dans mon travail sur la globalisation : il s'agit des implications (1) du ré-ordonnancement de l'espace-temps vécu et «imaginé » par les groupes humains, (2) de l'usage des nouvelles technologies de l'information et de la communication (NTIC) et (3) de l'interconnexion de ces deux aspects par l'avènement de modes de communication en réseaux. Je citerai aussi les éléments de définition proposés par Inda et Rosaldo [2002] parce que ces auteurs ont interrogé le terme selon une lecture plus spécifiquement anthropologique : la « globalisation » réfère à l'intensification des interconnexions à un niveau mondial, suggérant un monde qui bouge, se mélange, tisse des liens qui témoignent d'interactions et d'échanges culturels durables prenant la forme de mobilités complexes. Cette intensification génère des circuits d'inter- dépendance économiques, politiques, culturels et écologiques de l'échelle la plus locale à l'échelle globale. Le monde connaît une accélération radicale des flux de sujets et d'objets, c'est-à-dire de capitaux, personnes, biens, images et idées produisant alors un réagencement de l'espace et du temps. Dans ce contexte, la recherche pharmaceutique, la production industrielle des médicaments et les réseaux de commercialisation se sont déplacés ou ont émergé dans d'autres parties

* Anthropologue à l'IRD, UMI 233 TransVIHMI, INSERM U1175, Université de Montpellier, équipe Cultures locales et santé globale. 
1. Recherche conduite dans le cadre du programme en sciences sociales IRD-PUMC (Peking Union Medical College)/CAMS (Chinese Academy of Medical Sciences) (2006-2011) "Shift in Health Policies, Social Response and the Cultural Politics of Scientific/Traditional Knowledge in AIDS Research, Treatment and Care" coordonné par l'auteure.

du monde. Les modes de distribution et d'accès à ces produits se sont diversifiés et leur circulation s'est accélérée.

La recherche visant à la mise au point de médicaments pharmaceutiques indus- triels à partir de produits de la pharmacopée chinoise est née en Chine dans les années 1920 [Hoizey, Hoizey, 1988 ; Unschuld, $1986^{2}$ ]. Ces recherches portent aujourd'hui leurs fruits avec un développement sur presque un siècle. Résultant d'une volonté politique affirmée des années 1950 jusqu'à nos jours, ces politiques de recherche et de développement relèvent d'une idéologie moderniste de construction d'une science nationale dans le cadre d'un nouvel État-nation. La stratégie de la Chine populaire était de conserver une position relativement indépendante de la ronde des nations puissantes, reflétant la mise en œuvre du slogan maoïste bien connu « compter sur ses propres forces ». Pour discuter de la recherche pharmaceutique chinoise, ce texte s'appuiera principalement sur l'exemple du développement et de la circulation des combinaisons thérapeutiques à base d'artémisinine (CTA). Bien que l'artémisinine ait été découverte en 1972, plus de trente ans se seront écoulés avant la reconnaissance de son efficacité à une échelle mondiale par une recommandation de l'OMS [2004] pour le traitement du paludisme [Duffy, Mutabingwa, 2004 ; Woodrow, Haynes, Krishna, 2005]. Les CTA sont aujourd'hui utilisés massivement dans de très nombreux pays en développe- ment affectés par cette maladie. Cependant, une ombre assombrit ce succès avec l'émergence de résistances confirmées dans une zone à la frontière du Cambodge et de la Thaïlande. Ces résistances s'expliquent notamment par la pression médicamenteuse sur les vecteurs due aux insecticides et par le changement de comportement de ces vecteurs généré par l'usage des moustiquaires imprégnées. En janvier 2011 est lancé le Plan d'action mondial pour l'endiguement de la résistance à l'artémisinine (Global Plan for Artemisinin Resistance Containment) [OMS, 2011]. Si l'efficacité de ces traitements était remise en question, la majorité des pays en développement affectés par le paludisme n'aurait aucune alternative et la 
situation deviendrait très préoccupante. Il convient de préciser tout de même que le nombre d'infections par le paludisme a régressé dans le monde ces dernières années grâce à la mise au point d'une méthode de diagnostic rapide, aux CTA et aux moustiquaires imprégnées à longue durée d'action.

À l'image des dérivés de l'artémisinine, d'autres « nouveaux produits pharmaceutiques », se situant à la croisée de la biomédecine et de la médecine chinoise, sont prescrits en médecine chinoise intégrée ${ }^{3}$ en Chine dans le cadre du système de santé publique tout en entrant dans des réseaux de circulation à plusieurs échelles, locale, nationale ou internationale. Mais le fait qu'ils trouvent place dans la sphère de la « santé globalisée » par une réappropriation en contextes, dépend de plusieurs facteurs et n'est pas systématique. C'est le cas des dérivés d'artémisinine, à la différence d'un second produit qui sera présenté en contrepoint, la

2. Pour une histoire à mémoire longue des produits pharmaceutiques en Chine.

3. Résultat d'une insertion initiée après 1949, planifiée par des politiques publiques volontaristes : système de «médecines intégrées » produit par cette restructuration du système de santé sur un long terme.

71

tablette herbale Tang (Tang Cao Pian), préconisée en complément des traitements antirétroviraux du VIH et du sida en Chine dans le cadre du programme national de prise en charge depuis l'obtention en 2004 de son Autorisation de mise sur le marché (AMM) auprès de l'Agence nationale de sécurité sanitaire et alimentaire (State Food and Drug Administration : SFDA). Mobilisant ces deux traitements - les CTA et la tablette herbale antiVIH - l'article cherchera à répondre à une double question : quelle est la spécificité de la recherche et du développement de nouveaux produits pharmaceutiques en Chine ? Comment penser la rencontre de plusieurs paradigmes dans le champ de la pharmacopée et dans quelles mesures ces produits peuvent-ils être qualifiés d' « objets hybrides » résultant du nouage de " réseaux longs »? : ces deux notions empruntées à Latour [1991] seront interrogées dans une seconde partie. De tels réseaux présentent un intérêt pour comprendre les assemblages hétérogènes qui se constituent dans le champ de la pharmacopée et les multiples enjeux qui les animent. 
Le constructivisme social, courant fondateur du champ interdisciplinaire des Science and Technology Studies (STS), né au sein du Massachusetts Institute of Technology (MIT), inspire l'orientation théorique du texte. Rappelons que l'ensemble des normes, des principes, des concepts et des valeurs spécifiques aux savoirs et aux croyances identifiées dans un espace spatio-temporel donné produit un «style de pensée » spécifique et que la pratique scientifique peut alors être interprétée comme une pratique culturelle [Fleck, 2005]. Parmi les membres du groupe à l'origine des STS, incluant également Latour et Woolgar, plus connus dans la recherche francophone, Lakoff [2008] a initié des études sur le médicament envisagé comme technologie et sur les enjeux complexes dont il est l'objet : une « raison pharmaceutique » s'inscrit en filigrane le long du chemin qu'il par- court et qui conduit sur les traces de sa « vie sociale » au temps de la santé globalisée. Elle apparaît comme un fil conducteur qui sous-tend les processus d'imbrication mutuelle de la science, des règlementations et du marché dans la circulation des produits pharmaceutiques rendus possible grâce à l'émergence d'un marché mondialisé de la santé : une telle imbrication sera vérifiée une fois encore dans notre étude.

L'article est organisé en deux sections principales : la première porte sur la recherche et le développement de nouveaux produits pharmaceutiques à partir des exemples des CTA et de la tablette herbale anti-VIH ; la deuxième s'attache à discuter de la définition d'un nouveau produit selon des perspectives - etic (ma propre analyse) et - etic locales (celle d'acteurs chinois) et dans quelle mesure ces produits peuvent être qualifiés d'objets hybrides par l'identification de réseaux longs dans le sillage de Latour [1991]. La méthodologie de l'étude repose sur une recherche documentaire et sur une observation de cas de recherche et de développement en Chine portant sur les traitements innovants du VIH, en biomédecine, y compris en matière de développement vaccinal dans les années 2000, et en médecine chinoise (20062011, voir note 1) ; cette recherche a été étendue à d'autres produits qui entraient dans la composition de ces « nouveaux médicaments » potentiels issus de la pharmacopée traditionnelle ou populaire et de sa 
rencontre avec la biomédecine. Des entretiens ont été conduits avec un ensemble d'acteurs qui jalonnent la «vie » de ces médicaments, et dont les dynamiques relationnelles, ainsi que les stratégies, éclairent les enjeux qui nous intéressent : des acteurs de la recherche (directeurs de laboratoire, chercheurs, universitaires, techniciens), des acteurs médicaux (personnels de santé de divers échelons de la hiérarchie administrative hospitalière, personnels de cliniques privées, vendeurs de médicaments, pharmaciens et autres personnels exerçant dans les pharmacies des hôpitaux, des centres de santé et des pharmacies de détail), des acteurs officiels (cadres de santé de divers échelons, membres du ministère de la Science et technologie et du ministère de la Santé), enfin des acteurs de la société civile (organisations sociales, personnes vivant avec le VIH au titre de leur engagement dans un cadre associatif ou officiel, ou encore à titre personnel). Des observations d'essais cliniques et de cas de $R \& D$ ont eu lieu dans la mesure du possible, pour obtenir des données qui offrent un contrepoint à l'analyse de discours.

J'ai aussi collecté de nombreuses informations lors du $1^{\text {er }}$ Congrès mondial de l'Organisation mondiale de la santé (OMS) sur les médecines traditionnelles qui s'est tenu à Pékin du 7 au 9 novembre 2008. Concernant l'artémisinine et ses dérivés, l'équipe du laboratoire de l'institut de Médecine tropicale de l'université de Médecine Chinoise de Canton (Guangzhou zhongyi yao daxue) m'a donné accès à un certain nombre de documents. Ce laboratoire avait contribué à la découverte de l'artémisinine en menant des recherches cliniques dès les années $1970[\mathrm{Li}, 1990]^{4}$.

\section{Recherche \& développement et usages de nouveaux produits}

Le cas des combinaisons thérapeutiques à base d'artémisinine et la tablette herbale Tang

Les traitements à base d'extraits d'Artemisia annua L. (Qinghao) furent décou- verts en Chine populaire dans les années 1970. L'artémisinine (Qinghao su : extrait «d'herbe verte » en chinois) a été isolée par les chercheurs chinois en 1972 (résultat fructueux du premier projet national contre le paludisme, projet 523,1967$)^{5}$, et sa structure connue en $1979^{6}$. Le prestigieux prix Lasker-De Bakey de recherche clinique médicale a été attribué à Tu Youyou et son équipe en $2011^{7}$ 
4. Ces nouvelles données ont été collectées lors d'une recherche en cours portant sur l'histoire et l'anthropologie des traitements du paludisme en Chine et en Asie du sud-est dans le cadre de ma participation au programme ANR SOREMA (Sociétés, Résistances, Malaria), 2012-2015, porté par l'UMR 912.

5. Voir protocole et résultats de ce projet dans Tu [2011].

6. Voir les schémas des structures de l'artémisinine et des molécules dérivées (Dihydroartemisinin, Artemether, Arteether, Artelinic acid, Artesunic acid) dans van Agtamael et al. [1999, p. 199, figure 1] et $\mathrm{Tu}[2011$, p. 1218, figures $3 \mathrm{a}$ et b].

7. Le prix Lasker en sciences fait partie des plus prestigieux dans le monde. Depuis 1945, des scientifiques, des médecins et des fonctionnaires dont la contribution est reconnue comme majeure en matière de diagnostic, de traitement ou de prévention des maladies humaines, sont distingués par ce prix qui présage souvent d'une future sélection par le Comité Nobel. Le programme Lasker est considéré comme l'équivalent américain du Nobel. Le prix Lasker-DeBakey de recherche médicale clinique récompense des chercheurs

pour leurs travaux menés depuis les années 1970 ayant conduit à la découverte de l'artémisinine. Il convient ici de mentionner que la recherche fondamentale a été menée en Chine [Tu, 2011] et les essais thérapeutiques en Chine et en Tanzanie [Hsu, 2002]. Les botanistes avaient identifié Qinghao comme Artemisia apiacea et Huanghao hua comme Artemisia annua L. [Hsu, 2006, p. 506]. L'artémisinine peut être extraite de trois espèces d'Artemisia : elle est présente en quantités impor- tantes dans l'armoise Artemisia annua L. et en quantités réduites dans Artemisia apiacea et Artemisia lancea [Wright et al., 2010].

Dans une première phase, l'artémisinine fut mise au point pour un usage en monothérapie et diffusée sous cette forme comme traitement antipaludique en Chine et en Asie du sud-est dans les années 1980 et $1990^{8}$. Dans une deuxième phase, les polythérapies ont été testées avec succès et promues par l'OMS dans les années 2000. Ces nouveaux traitements permettent d'éliminer rapidement le parasite grâce à l'artémisinine puis d'éviter une ré-infestation du malade grâce au produit biomédical antipaludique de synthèse associé (chloroquine, sulfadoxine- pyriméthamine, méfloquine, luméfantrine, amodiaquine).

Ce qui fait la singularité de la démarche de recherche de Tu, notamment si on la compare avec celle utilisée pour la tablette herbale Tang, est la référence 
directe à la pharmacopée ancienne comme source d'inspiration et comme point de départ de l'expérimentation [Tu, 2011]. Tu et d'autres chercheurs chinois ont publié une ancienne série d'articles dans la prestigieuse revue de science Nature en 2011, dans un numéro spécial sur les médecines traditionnelles asiatiques [Coll., 2011]. Le récit par Tu de sa découverte dans ces publications n'échappe pas à des aspects qui relèvent de la construction sociale et idéologique de la science, pour au moins deux raisons. Premièrement, cette revue est un dispositif médiatique qui contribue à façonner la science. Deuxièmement, la Chine donne dans ses discours officiels une place importante à la construction d'une science nationale qui transparaît dans les publications scientifiques. Le discours officiel mobilise des idées et des pratiques de la recherche pour valoriser un exemple paradigmatique qui montre les avancées de la science « chinoise » à la fois en médecine traditionnelle par les références à des textes anciens issus de la « grande tradition écrite chinoise » et en biomédecine par l'usage de la méthode Evidence Based Medicine (EBM) ${ }^{9}$. Dans le cas de l'artémisinine, où le récit se situe-il entre

qui ont contribué avec brio à l'amélioration du traitement clinique des patients, http://www.laskerfoundation.org/awards/index.htm (page consultée le 20 septembre 2012).

8. Des informations complémentaires seront recueillies à ce sujet lors d'une enquête en Chine prévue en 2013.

9. L'EBM est définie comme « l'usage consciencieux, explicite et judicieux des données actuelles les plus probantes dans la prise de décision sur le soin des patients individuels » [Sackett et al., 1996, p. 71]. La méthode EBM tire son origine de travaux en agronomie dans les années 1920 : Fisher conduisit des tests d'inférence et des expérimentations contrôlées et le lien entre agronomie et médecine a été clairement établi [Fisher, Yates, 1938]. Cette méthode d'établissement de la légitimité suscite cependant des controverses : l'expérience clinique est délégitimée au profit d'une médecine régulatoire, fondée exclusivement sur la rationalité scientifique dans une période de réductions budgétaires et d'atteinte à la liberté clinique [Sackett et al., 1996], dont la légitimité repose sur des essais cliniques concluants. Selon le Comité international des rédacteurs de revues médicales, un essai clinique est un «projet de recherche qui affecte de

74

une histoire « officielle » et une histoire « factuelle»? Les conditions de production du savoir scientifique révèlent une recherche « située » socialement : la recherche sur l'artémisinine s'inscrit dans le cadre socio-historique du retour à une interprétation de textes anciens. Tu, principale investigatrice de cette équipe de recherche, s'est penchée sur ces textes et sa réflexion a 
conduit à repenser un protocole infructueux conduit dans le cadre d'un paradigme très normatif avec son cortège de procédures qui encadrent strictement l'activité de recherche. À partir d'une recherche documentaire dans les traités médicaux ou de materia medica classique, elle s'intéresse aux usages décrits dans le traité de Gehong ${ }^{10}[\mathrm{Tu}, 2011]$. Pour elle, l'objectif était d'avancer face à un échec antérieur de l'expérimentation et d'élaborer un nouveau protocole : les observations s'étaient révélées non reproductibles alors que la reproductibilité est l'une des conditions nécessaires à la validation scientifique. Elle comprend alors que les composants actifs auraient pu être détruits par l'échauffement généré par le mode d'extraction moderne. En effet, la méthode décrite par Gehong consiste à extraire le jus de la plante fraîche ${ }^{11}$. Or la méthode moderne de préparation utilisée de nos jours en phyto-médecine, est la décoction et diffère notablement de la méthode ancienne. L'étude de la mise au point de l'artémisinine à partir des trois espèces d'armoise (Artemisia annua L., Artemisia apiacea, Artemisia lancea), des méthodes anciennes à l'établissement de la légitimité par la méthode EBM, et de leurs usages différenciés selon les périodes historiques, éclaire notre tentative de définition et de catégorisation des « nouveaux » produits. Les méthodes anciennes sont décrites dans des traités médicaux appartenant au corpus des pharmacopées historiques ${ }^{12}$ dont le plus ancien, qui remonte au IV ${ }^{e}$ siècle [Zhou hou bei ji fang par Wen, 1983], décrit l'usage thérapeutique de Qinghao pour traiter des fièvres épisodiques [Hsu, 2006]. Selon Tu [2011], les sources historiques qui mentionnent le Qinghao et ses indications thérapeutiques sont rares ; les usages thérapeutiques contre les fièvres concordent chez les deux auteures. Dans ce cas, nous n'observons pas de polysémie du remède et a fortiori il ne s'agit pas d'une panacée contrairement au cas du ginseng évoqué ci-après. À partir de la comparaison des concentrations de la substance active (artemisinine) par les méthodes anciennes et par les méthodes actuelles de préparation, l'étude de Wright et al.

façon prospective des sujets humains à des groupes d'intervention et de comparaison afin d'étudier la relation de cause à effet entre un acte médical et l'évolution d'un état de santé » (cité par Labrousse, $[2010$, p. 6]). Un traitement de contrôle, souvent un placebo, des mesures spécifiques de résultats visant à évaluer le traitement testé, et une méthode fondée sur l'assignation aléatoire d'inclusion des patients sont les condi- tions de recherche qui caractérisent les essais cliniques randomisés [Labrousse, 2010].

10. Voir les usages décrits dans Zhou hou bei ji fang, le manuel de prescriptions d'urgence par Gehong, version de la dynastie Ming (XVI siècle) par Wen [1983]. 
11. Pour plus de détails sur les méthodes d'extraction ancienne, voir Hsu [2006].

12. Le Shennong bencao jing (Traité de pharmacopée de Shennong) de la dynastie des Han (Ier siècle ère chrétienne) et le Bencao gangmu (Compendium de matière médicale) attribué au célèbre médecin chinois Li Shizhen (XVI siècle, dynastie des Ming) sont les pharmacopées historiques officielles les plus reconnues. Shennong, l'un des empereurs mythiques fondateurs de la civilisation chinoise, est aussi la divinité de la médecine et de l'agriculture. Sont identifiées aussi d'autres pharmacopées qui reflètent la diversité des cultures locales chinoises ou d'autres groupes ethniques. Depuis 2010, la médecine chinoise est inscrite au patrimoine immatériel mondial de l'Unesco.

$\overline{75}$

[2010] démontre que l'efficacité optimale contre le paludisme est obtenue par les méthodes anciennes, c'est-à-dire par l'extraction du jus de la plante fraîche plutôt que par la décoction de la plante sèche, indiquée aujourd'hui en Chine et ailleurs notamment sur les sites internet. Les deux manières d'extraire présentent une efficacité potentielle mais le jus obtenu avec la plante fraîche contient une dose suffisante d'artémisinine pour éradiquer le parasite lors de l'expérimentation animale conduite sous la forme d'essais précliniques.

Considérons à présent la mise au point de la tablette herbale Tang et l'élaboration de protocoles intégrant l'astragale et ses dérivés. Des compositions à base de plantes sont testées depuis les années 1990 selon des procédures de recherche clinique en Chine, en Afrique (Tanzanie) et au niveau de la recherche internationale en vue de la mise au point de traitements du VIH et du sida. Lors d'une étude précédente sur la recherche médicale en médecine chinoise intégrée visant au traitement des maladies chroniques [Micollier, 2010], et plus spécifiquement, de l'infection par le VIH et des cancers, j'avais sélectionné plusieurs plantes de la pharmacopée. Une recherche documentaire sur les résultats d'essais cliniques publiés dans des revues chinoises ou internationales m'avait permis d'identifier trois plantes Radix Ginseng, Radix Astragali, Radix Glycyrrhizae en fonction du critère de leur récurrence dans les essais cliniques conduits en Chine et éventuellement en coopération de recherche internationale depuis une vingtaine d'années ${ }^{13}$. Ces plantes d'origine locale étaient parfois des panacées. Leurs usages thérapeutiques sont alors décrits dans plusieurs pharmacopées historiques : c'est le cas du ginseng qui a traversé l'espace-temps thérapeutique - depuis la pharmacopée chinoise ancienne et ses usages populaires et spécialisés jusqu'à sa diffusion récente sous différentes formes empruntant les canaux de diffusion 
de la globalisation (NTIC). La propriété de ces plantes, retenue ces dernières années, parmi leurs multiples usages traditionnels et populaires, est l'action immuno-stimulante ou immuno-régulatrice. Les essais cliniques portant sur la tablette herbale Tang ont été conduits au début des années 2000 à l'hôpital You'an, l'un des principaux hôpitaux de Beijing assigné à la prise en charge $\mathrm{du} \mathrm{VIH}$ et au traitement des maladies opportunistes, plus spécifiquement les hépatites en ce qui concerne cet hôpital. Son usage en traitement complémentaire aux antirétroviraux (ARV) s'est progressivement répandu dans les provinces dans le cadre du système de santé publique ${ }^{14}$. Ceci dit, son utilisation n'est pas bien acceptée dans certains hôpitaux par les acteurs du soin ou de l'administration sanitaire qui doutent de son efficacité. De plus, la prescription de la tablette Tang représente un coût supplémentaire. En effet, les hôpitaux chinois commercialisent eux-mêmes la plupart des médicaments prescrits par les cliniciens travaillant dans leurs services alors que dans ce cas ils doivent faire appel à la compagnie pharmaceutique qui détient le brevet et a obtenu l'AMM.

\section{Références de ces publications dans Micollier [2011].}

14. Pour des exemples en contextes de ses usages et de sa perception par les patients, par le personnel de santé et par des acteurs officiels, voir Micollier [2009b ; 2011].

76

Des processus d'innovation complexes qui bousculent les catégorisations emic du médicament

Par la découverte de l'artémisinine, la contribution de Tu est un exemple réussi de développement de médicaments industriels qui tirent leur origine de la médecine chinoise ${ }^{15}$. Comme nous l'avons vu, l'artémisinine a bien été « découverte » alors que la tablette Tang a été «mise au point ». Cette différence, entre ces deux chemins qui conduisent à l'avancée scientifique dans ce domaine, souligne l'importance des processus de recherche en pharmacologie et contribue à éclairer le questionnement sur les nouveaux produits.

Dans le contexte de la recherche en médecine chinoise en Chine visant à un alignement sur les normes scientifiques internationales par la validation EBM, les produits des pharmacopées historiques sont traités par les chercheurs qui 
les manipulent comme des produits pharmacologiques biomédicaux : n'apparaît alors aucune référence aux savoirs qui sous-tendent les usages thérapeutiques des plantes fondés sur une expérience clinique et des procédures antérieures à l'exception de la mention de la première source d'identification des plantes souvent dans les pharmacopées historiques qui n'est d'ailleurs pas systématique.

La question de la construction sociale de la légitimité se pose avec d'autant plus d'acuité dans la recherche à la frontière de deux ou plusieurs paradigmes : elle mobilise au moins deux théories de la connaissance et deux systèmes de production des savoirs médicaux, ceux de la médecine internationale contemporaine (biomédecine) et ceux de la médecine chinoise contemporaine. Dès lors, quelles théories et catégorisations sont porteuses et productrices de sens ? La biomédecine ou la médecine chinoise, la médecine conventionnelle internationale ou bien les médecines non conventionnelles (CAM) ou encore, audelà de ces références, un autre modèle qui rendrait caduques ces distinctions si nous nous attachons à l'étude pratique des « nouveaux produits », de leur mise au point, de leurs usages et de leurs conditions de diffusion ? Les facteurs de légitimation relèvent soit de la méthode pré-EBM, à savoir l'étude empirique de cas cliniques produisant un savoir préservé dans les traités médicaux et fondé sur leur interprétation par des institutions et des praticiens, soit de la méthode EBM. Or le terme evidence renvoie à la preuve empirique, à l'appartenance à des registres et aux procédures de hiérarchisation des preuves. La biomédicalisation progressive de la médecine chinoise est attestée par l'utilisation de la méthode des essais cliniques et l'usage de plateformes mobilisant la clinique et le laboratoire [Micollier, 2007 a, b ; 2009 a, b ; 2011]. Le statut épistémologique de cette méthode résulte d'une hybridation entre biologie et

15. Un autre exemple remarquable est celui de la mise au point de l'Arsenic trioxide par Zhang Tingdong de l'université de Harbin. Ce produit a obtenu une AMM délivrée par la Food and Drug Administration (FDA) des États-Unis pour le traitement de la leucémie en 2000. Zhang a apporté une contribution unique au développement de ce médicament en réussissant à séparer un constituant toxique du complexe thérapeutique. 
médecine qui suppose de repenser, d'une part les relations entre la clinique et le laboratoire ${ }^{16}$, d'autre part les relations entre le normal et le pathologique.

Les difficultés à classer ces produits apparaissent de manière évidente aussi dans le simple fait que la médecine chinoise est une médecine conventionnelle en Chine puisqu'elle est normalisée et intégrée au système de santé publique depuis les années 1950. En outre, mise à jour tous les deux ans et enregistrée à l'OMS depuis 1992, la pharmacopée chinoise contemporaine officielle [OMS, 2010] intègre tous types de traitements quelles que soient les connaissances mobilisées pendant le processus de R\&D ${ }^{17}$. Cette recension de référence réglementaire et normalisée établit l'inventaire des médicaments de médecines de Chine et de biomédecine qui y apparaissent en proportion quasiment identique. En outre, y sont répertoriés les complexes médicinaux (compounds) de materia medica (zhongyao) ainsi que des complexes « innovants » à condition d'être validés selon les normes légales chinoises, par l'obtention de l'AMM délivrée par la SFDA. Dans la mesure du possible, ces complexes nouveaux sont traités et testés selon les mêmes procédures de pharmacologie clinique que les médicaments biomédicaux. Les CTA et la tablette Tang sont recensés dans la pharmacopée contemporaine. Les CTA connaissent un succès paradigmatique pour des médicaments industriels issus de médecines traditionnelles en santé globalisée alors que la tablette Tang est passée inaperçue au-delà des frontières chi- noises. Un tel intérêt pour l'artémisinine est sans doute comparable au cas de l'aspirine au xx siècle. Précisons tout de même que cet extrait actif n'est pas perçu par les médecins chinois comme une médecine traditionnelle mais comme un produit biomédical [Hsu, 2009].

Nos deux études de cas sont envisagées selon divers angles d'approche, la mise au point par la recherche, les usages, la diffusion : les mêmes produits peu- vent être qualifiés d' «intégrés», de «combinés», d' «émergents» ou bien d' « hybridés » de mon point de vue en tant que chercheure qui travaille sur l'intérêt de cette terminologie, un tel point de vue - etic croise alors des points de vue - etic locaux explicités par la suite. Plusieurs qualifications conviennent pour le même produit. Un objet hybride est complètement autre par nature en tant que résultat, alors que la qualité $d$ ' « hybridé » réfère seulement à une modalité du produit. Dans le premier cas, un ensemble de 
traitements - divers CTA, à base d'extraits d'un produit de la pharmacopée historique - est associé avec un produit biomédical : il peut donc être qualifié de traitement combiné. Dans le second cas, il peut aussi être considéré comme « combiné » par ses usages thérapeutiques, en sa qualité de traitement complémentaire aux ARV. Par ailleurs, il s'agit d'un complexe de phytomédecine, une tablette herbale aux douze ingrédients actifs

16. Un réalignement entre la clinique et le laboratoire s'opère avec des plateformes biomédicales « normalisées » qui intègrent systématiquement les innovations techniques [Cambrosio, Keating, 2003].

17. Précisons tout de même qu'en Chine, la mémoire écrite et orale est héritée d'une longue histoire et d'une grande diversité des cultures locales et recèle d'autres pharmacopées dont les produits sont ou seront intégrés à la pharmacopée chinoise contemporaine officielle.

78

provenant de plantes de la pharmacopée locale ${ }^{18}$. La théorie, la méthode et le régime de la preuve qui ont conduit à sa mise au point relèvent de la biomédecine. Le complexe appartient donc à la catégorie des traitements intégrés. Il peut aussi être classé dans la catégorie « combiné » par ses usages thérapeutiques en sa qualité de traitement complémentaire aux ARV.

À partir de seulement deux exemples, nous avons souhaité expliquer comment le choix de catégories génériques pour nommer les nouveaux produits (combinés, intégrés, émergents, hybridés) est rendu caduque par des modalités qui complexifient la catégorisation et confirment une appartenance à plusieurs catégories.

Ces deux produits, CTA et tablette Tang, sont également « émergents » (nouveaux) et «hybrides », relevant d'une médecine intégrée car ils ont été mis au point par la méthode scientifique expérimentale et conforme aux procédures de validation EBM. Ils peuvent aussi être considérés comme adaptés de la materia medica ou innovants.

\section{Définitions - etic des nouveaux produits : objets hybrides et socialité en réseaux}

La recherche clinique dans ce contexte vise soit, à l'adaptation ou à l'actualisation de traitements indiqués dans des pharmacopées avec de nouvelles avancées de la connaissance, soit à la mise au point ou à la découverte de 
traitements innovants, c'est-à-dire issus de la materia medica mais dont la (re)composition est nouvelle. Le produit se présente alors comme un nouveau complexe (compound). À la lumière de cette distinction, les cas des traitements à base d'artémisinine et ses dérivés et du complexe herbal (tablette Tang) permettent de souligner la difficulté à définir et classifier ces produits. Les deux produits constituent l'un et l'autre des traitements innovants : l'artémisinine est une découverte, la tablette herbale est le résultat d'une mise au point. Ces produits peuvent aussi être considérés comme des adaptations de traitements par la généalogie qui nous mène à des sources anciennes telles que les pharmacopées historiques constituées de traités médicaux et de la materia medica, jusqu'aux pharmacopées contemporaines, la plus récente ayant été enregistrée à l'OMS en 2010.

Le statut épistémologique diffère en fonction de leur constitution : pour le premier, un extrait de plante de la pharmacopée traditionnelle est produit industriellement : la molécule active a été clairement identifiée et son dosage étudié (artémisinine) associé à un médicament de synthèse. L'action de l'artémisinine et de ses dérivés permet d'éradiquer le parasite en quelques jours et celle du médicament biomédical prend le relais afin d'éviter la réinfestation ${ }^{19}$. Les CTA sont

18. Herba Erodii/Herba Geranii ; Flos Lonicerae ; Herba Moslae ; Radix Astragali ; Flos Gossam- pini ; Pericarpium Trichosanthis : Radix Bupleuri ; Herba Hedyotis Diffusa ; Radix Glycyrrhizae ; Caulis Spatholobi ; Herba Solani Nigri ; Radix Oryzae Glutinosae [Business/Finance China, 2009].

19. Les traitements d'artéminisine en monothérapie ne sont plus recommandés aujourd'hui au regard des avancées de la science et de la confirmation de résistances au traitement.

\section{9}

sans aucun doute des nouveaux produits parce qu'ils associent l'artémisinine et ses dérivés ainsi qu'un produit de synthèse, en général un médicament qui n'est pas extrait d'une plante sauf un, la quinine, extrait actif d'écorce de quinquina. De plus, l'action des deux produits est décalée dans le temps introduisant une modalité spécifique de l'efficacité. Toutefois, l'artémisinine et ses dérivés sans l'association du médicament de synthèse relèvent aussi de l'innovation : l'artémisinine est un extrait actif de plantes de la pharmacopée historique chinoise validé par la méthode EBM.

Pour définir un nouveau produit, de nombreuses distinctions et typologies ont 
été proposées par les chercheurs chinois qui se sont penchés sur la question de la rencontre des paradigmes entre science occidentale et savoirs traditionnels depuis l'introduction de la médecine occidentale en Chine au XIX ${ }^{\mathrm{e}}$ siècle, nous offrant ainsi un point de vue - etic inscrit dans le contexte local. Les industriels s'intéressent aussi à cette question dans le cadre du développement des produits. À ce propos, le premier congrès mondial sur la médecine traditionnelle organisé par l'OMS, qui a eu lieu à Pékin en 2008, s'est avéré un événement symbolique fort marquant la reconnaissance institutionnelle des médecines traditionnelles à une échelle globale. Dans les communications du congrès, de nombreux intervenants avaient des intérêts économiques ou politiques à défendre pour la promotion des nouveaux produits pharmaceutiques industriels issus d'une manière ou d'une autre des pharmacopées historiques.

Par exemple, Hu [2008], président d'un groupe pharmaceutique chinois (Zhejiang Conba Pharmaceutical Co., Ltd), a proposé une distinction assez répandue entre « materia medica traditionnelle »(CMM : Chinese Materia Medica) et " médicaments botaniques modernes », terme que je vais expliciter. Une telle intervention témoigne de l'influence très directe des lobbies pharmaceutiques chinois (et internationaux) dans le financement, la participation, la publicité et la vente de produits et de technologies in situ. $\mathrm{Hu}$ [2008] conclut qu'une stratégie de développement de l'industrie de la materia medica chinoise consiste à trouver une nouvelle identité ou bien une nouvelle forme qui attire graduellement l'attention d'un public élargi sous l'appellation de «médicaments botaniques modernes ». La matière médicale chinoise peut facilement, sous cette nouvelle forme qui transforme son identité, entrer dans le marché pharmaceutique domestique et international. Hu introduit une différence de nature entre les deux catégories alors que nous savons que la majorité des médicaments industriels ont une origine naturelle : notons cependant que l'une se transforme dans l'autre au cours du processus de recherche et de développement du médicament et dans le cadre de ses circulations sur le marché international, par les réappropriations en contextes. Il compare les processus de recherche et de développement des deux types de produits et insiste surtout sur les avantages des "médicaments botaniques modernes », tout en les enracinant au niveau symbolique et identitaire dans la materia medica historique.

Rappelons que la «médecine chinoise » (zhongyi) n'avait pas été institution- 
nalisée dans le cadre des structures de santé publique dans les années 1920, date

de la création d'un système de santé publique par la République de Chine (1911-1949) avec la création du premier ministère de la Santé en 1928 ; elle avait été abandonnée au profit de la «médecine occidentale » (xiyi), introduite au XIX ${ }^{\circ}$ siècle. En contraste, le nouvel État, la République Populaire de Chine, qui naît en 1949 suite à la deuxième révolution chinoise du Xx siècle, va faire preuve d'une volonté politique forte en matière de politiques publiques de santé en intégrant la médecine chinoise dans le système de santé publique et en assurant sa promotion. Taylor [2005] identifie trois phases successives en matière de politiques publiques et de transmission : une période de coopération (1945-1950), d'unification (1950-1958) et d'intégration (1958années 2000). Hsu [2008] propose une périodisation en deux phases en ajoutant une interprétation de la transformation : de 1958 à la fin des années 1980, est née une tradition inventée de « médecine traditionnelle chinoise » en référence au concept de Hobsbawm et Ranger [1983], et de 1990 à nos jours émerge une tradition "marchandisée » et une autre modernité scientifique, avec le développement de la "médecine chinoise et de la pharmacothérapie » (zhongyiyao xue). Des éléments de la terminologie vernaculaire, tirant leur origine de la période d'élaboration et de mise en œuvre des politiques maoïstes ${ }^{20}$, apparaissent comme une métaphore pouvant éclairer les significations et la conceptualisation locales de cette rencontre qui s'est produite dans le cadre d'un nouvel État-nation. Les termes d'antériorité et de postériorité «d'abord la médecine chinoise ensuite la médecine occidentale» (xianzhong houxi) ou l'inverse (xianxi houzhong), «d'intégration » (jiehe), « d'unification » (tuanjie), de « coopération » (hezuo) sont utilisés pour définir cette « nouvelle médecine » dans les discours officiels et les sources médicales. Enfin, dans les années 2000, émerge la notion de «synthèse » (zonghe) entre les deux médecines [de Bruyn, Micollier, 2011].

Concernant l'étude portant sur les processus de production de savoirs culturels « hybrides » documentés avec l'exemple paradigmatique des savoirs thérapeutiques [Micollier, 2011], j'avais conclu qu'il était inapproprié d'utiliser ce concept dans le sillage de Latour, même de manière opératoire 
et/ou méthodologique ; son usage m'était apparu a fortiori exclu d'un point de vue épistémologique. Je me référais alors à l'usage d'hybride dans le sens de Scheid [2002] et de Frank et Stollberg [2004] : le premier définit l'hybridation comme synthèse en contraste avec l'intégration ; les seconds distinguent un processus d'hybridation structurelle, produisant des formes stables relativement pérennes à terme, d'un processus d'hybridation conjoncturelle produisant des formes instables destinées à disparaître lors de l'action de recomposition continue qui est à l'œuvre.

Les travaux que je mène sur la pluralité thérapeutique, sa conceptualisation, ses mécanismes d'application et ses voies de légitimation, me conduisent à distinguer clairement les concepts « hybridité/hybridation » et « d'objet ou de tech- nique hybride $»$.

20. 1945-1963 pour la médecine [Taylor, 2005].

81

Un objet hybride est complètement autre par nature, en tant que résultat, alors que la qualité d' "hybridé » réfère seulement à une modalité du produit. L'étude spécifique de l'usage de certains ingrédients plutôt que d'autres dans les protocoles de recherche et de l'inscription de ces substances dans les pharmacopées locales peut éclairer les processus de production et de diffusion des savoirs thérapeutiques «émergents » ou hybrides adaptés localement. Ces processus révèlent une interprétation singulière des rapports entre savoir thérapeutique empirique et savoir savant ainsi que des rapports entre les savoirs « savants traditionnels » (chuandong xueshu) et « populaires » (minjian) [Micollier, 2011].

Les nouveaux produits peuvent-ils être considérés comme des «objets hybrides» au sens de Latour [1991]? L'analyse d'une socialité en réseaux (réseaux longs) en lien avec la production d'objets hybrides proposée par Latour [op. cit.] a la fonction heuristique de nous éloigner de l'analyse systémique. C'est pourquoi même si l'hybridation de systèmes ou de savoirs médicaux est envisageable, s'inscrit toujours en filigrane le même type de rationalité consistant à « faire système » c'est-à-dire que la recherche et l'identification d'une rationalité de la nouvelle configuration inter- et intrasystèmes apparaissent encore comme une démarche cohérente. Le concept 
d'hybridité selon Latour n'est justement pas synonyme de complexité, pluralité, syncrétisme. Un « système hybride » est une association de termes paradoxale car la qualité d' « hybride » ou l'action d'hybridation sont par définition peu systémiques.

Dans le prolongement de cette réflexion, les termes «pratiques ou itinéraires hybrides » mis en évidence à l'aide d'une approche de facto systémique sont peu adaptés : il convient alors de conserver les qualificatifs « complexes, pluriels ou syncrétiques » utilisés communément dans la littérature académique sur la pluralité thérapeutique (et religieuse), dont l'usage est chronologiquement antérieur à celui d' « hybride » .

Dans le champ de la pharmacopée chinoise contemporaine, à partir de nos deux études de cas, se produit une action d'hybridation qui donne naissance effectivement à des « objets hybrides » par son articulation avec la théorie des réseaux de Latour et la possibilité d'identifier un "réseau long ». L'intervention d'un grand nombre d'acteurs, d'objets, de procédures, de régulations, de représentations et d'usages et leurs interactions depuis la conception jusqu'à la consommation d'un produit pharmaceutique construisent et nouent la « chaîne du médicament » [Garnier, Levy, 2007]. Cette chaîne opératoire peut être considérée comme une modalité significative d'un « réseau long » et le médicament comme un « objet hybride »: un tel objet est une construction innovante résultant de liens en réseaux de socialité et de processus de circulation et d'articulations entre idées, acteurs, usages ou techniques hétérogènes [Latour, 1991]. Il est donc le produit d'un processus d'hybridation identifiable en « suivant un réseau long ». Des assemblages hétérogènes construits par nouage d'objets de nature éclectique tels que les pratiques sociales et les ressources matérielles, l'association de ces deux objets étant fondamentale dans la théorie des réseaux de Latour, donnent à voir des liens entre

82

des interactions qui se produisent sur un continuum de l'échelle locale à l'échelle globale intégrant les niveaux intermédiaires et des relations entre les acteurs existant par ces processus. L'analyse des réseaux consiste donc avant tout à suivre les réseaux sur toute leur longueur et, appliqué à l'objet social et 
matériel «médicament », à suivre le « réseau long » porteur de sens pour comprendre la construction innovante.

L'identification, les modalités et la démarche de suivi des réseaux qui dessinent les contours de cette « chaîne » éclairent les divers enjeux qui se cristallisent autour des médicaments qu'ils soient scientifiques, sociaux, économiques, culturels ou éthiques. Par ailleurs, s'appuyant sur une lecture biographique de l'objet non humain, plus précisément sur le concept de «vie sociale des choses »d'Appadurai [1986], des anthropologues précurseurs dans le champ de l'anthropologie du médicament se sont intéressés à la « vie sociale » du médicament [van der Geest, Whyte, Hardon, 1996 ; Whyte, van der Geest, Hardon, 2002]. Ils ont analysé les produits pharmaceutiques comme des produits de consommation dans un contexte de marché globalisé de la santé : ces produits circulent dans le monde et sont projetés dans « la vie sociale » par les enjeux complexes dont ils sont l'objet et le nombre d'acteurs locaux et globaux qui les manipulent. Ils sont donc révélateurs de processus sociaux.

Suivons enfin le « réseau plus ou moins long » des « constructions innovantes - médicaments » que nous avons choisi comme étude de cas. L'objectif de la première section était de mettre en évidence dans le processus d'innovation connu des médicaments, c'est-à-dire dans le processus de transformation d'un traitement de médecine locale en médicament pharmaceutique classique, comment on passe du registre originel des médecines traditionnelles à celui de la biomédecine par la découverte ou la mise au point.

Revenons tout d'abord à l'exemple des CTA. Dans les années 1960 est constatée, partout dans le monde, l'émergence de résistances à la chloroquine, puis dans les années 1980 à la méfloquine. Enfin dans les années 2000, en l'absence de nouveau traitement biomédical qui ne générerait pas rapidement de résistances, les acteurs de la santé internationale s'intéressent à l'artémisinine. Dans les années 1980, des milliers de patients infectés par le paludisme ont été traités par l'artémisinine et ses dérivés en monothérapie (fabrication industrielle) en Chine. Le succès de ces traitements a attiré l'attention des chercheurs, des acteurs et des institutions de santé à un niveau international [Klayman, 1985]. Rappelons que la recherche a été conduite en Chine [Tu, 2011 ; Li, 1990] et en Tanzanie [Hsu, 2002]. Depuis les années 2000, l'usage en polythérapie est tout d'abord un succès en matière de 
«médecine combinée» par la prise différée de traitements issus d'extraits actifs de produits de la pharmacopée traditionnelle associés à un médicament biomédical. Enfin, c'est un exemple paradigmatique par sa circulation dans le monde qui lui donne une place privilégiée parmi ces produits de santé globalisée agréés par des institutions médicales locales, nationales et internationales. Le suivi du réseau long des CTA révèle la notoriété en retour de l'artémisinine et de la

83

plante Artemisia annua $L$. par la tisane qui est utilisée à l'époque contemporaine et vendue sur internet [Hirt, Lindsey, 2006]. Par ce médium, la référence aux sources est souvent « inventée » à des fins de légitimation et de promotion des produits. Le retour aux sources visant à trouver des indications dans la materia medica a été effectué dans le cadre académique de la recherche en médecine et en histoire [Tu, 2011; Hsu, 2010]. Les résultats de ces recherches ont justement permis de démontrer que l'usage le plus efficace est conforme à celui décrit dans les sources anciennes : la concentration de l'extrait actif est moindre dans la décoction de la plante sèche en comparaison avec le jus de plante fraîche. Une autre originalité de la nature du modèle CTA est la combinaison dans un même médicament d'une molécule de synthèse et d'une molécule naturelle.

Penchons-nous à nouveau sur notre deuxième étude de cas, qui porte sur la tablette herbale antivirale dont le destin n'a pas été le même que celui de l'artémisinine. La tablette a été testée, soit comme un produit alternatif/substitutif aux ARV, soit comme un produit complémentaire, un aspect d'ailleurs controversé qui soulève de nombreuses questions éthiques. Jusqu'en 2007, les protocoles et les politiques publiques dévoilent l'ambiguïté de l'objectif de la recherche conduite sur ce produit [Micollier, 2007b]. La position du gouvernement est clarifiée par le vice-ministre de la santé en charge de la gestion de la médecine traditionnelle, Cao Hongxin, en novembre 2008 lors du premier congrès mondial des médecines traditionnelles organisé par l'OMS et sponsorisé par de nombreux laboratoires pharmaceutiques chinois, qui confirme un usage thérapeutique complémentaire. L'espace limité de diffusion de la tablette Tang peut ainsi être rapporté au fait que les essais cliniques ont été menés exclusivement en Chine ${ }^{21}$, l'AMM accordée 
par la SFDA de Chine n'a pas été étendue par la validation institutionnelle d'instances internationales ; l'action antivirale du produit n'est pas reconnue en recherche internationale. Un certain nombre d'acteurs de soin et de cliniciens en Chine doutent de son efficacité. Sa mise au point dans les années 2000 est très récente et une relative opacité des objectifs de recherche dans les protocoles fut doublée de celle de la politique publique chinoise. Étant donné que les plantes à l'origine des nouveaux produits font partie de la pharmacopée locale, elles peuvent bénéficier a priori des connotations favorables associées aux produits médicaux alternatifs ou complémentaires (Complementary and Alternative Medicine) qui favorisent leur diffusion à un niveau international. La réappropriation locale est alors facilitée.

En Chine, ces produits sont fabriqués industriellement par des entreprises locales qui contribuent souvent à la recherche et à leur développement, et circulent ensuite dans le monde grâce aux réseaux de commercialisation qui se tissent dans le contexte actuel d'un marché globalisé de la santé. C'est ainsi que des espaces thérapeutiques complexes se construisent: ils offrent une palette variée de

21. Résultats de ces essais dans Shao [2008]. Zou et al. [2012] répertorient des recherches conduites selon les normes EBM et analysent des résultats d'essais cliniques testant des traitements de l'infection par le VIH de médecine chinoise. [Wang, Zou, 2007 ; Wang, Liang, 2011], de la même équipe de chercheurs de l'Académie nationale de médecine chinoise (Zhongguo zhongyi kexueyuan), dressent un état d'avancement des recherches de médecine chinoise sur le VIH et le sida.

84

médicaments d'origines hétérogènes qui s'enrichit à mesure que les produits traversent les frontières géographiques et temporelles. Nombre de sites internet assurent la promotion par la publicité et vendent des tisanes : la commercialisation en ligne de la tisane anti-paludisme est un exemple significatif ${ }^{22}$. L'usage d'internet facilite l'allongement des réseaux dans le temps et dans l'espace en démultipliant les acteurs. Les nouvelles technologies de l'information et de la communication (NTIC) jouent donc un rôle décisif dans l'émergence d'objets hybrides dans le cadre dynamique d'une circulation globale de ces objets thérapeutiques.

Pour la tablette herbale anti-VIH, la chaîne du médicament dans l'espace institutionnel s'est arrêtée à l'AMM de l'agence de contrôle sanitaire et 
alimentaire chinoise. L'espace de diffusion a pris la forme d'un réseau plus « court » géographiquement mais la recherche et le développement du produit dans l'industrie pharmaceutique ont pris celle d'un réseau « long » en matière de théorie des connaissances et de mobilisation de savoirs. Le réseau est de facto plus limité parce qu'il s'est arrêté aux frontières du pays qui l'a mis au point. Pourtant, entrent dans sa composition des extraits de plantes d'usages thérapeutiques très communs en médecine traditionnelle et populaire en Chine telles que Radix Astragali et Radix Glycyrrhizae. En contraste, le réseau est plus étendu dans l'étape fonda- mentale $R \& D$ de la « chaîne » du médicament: le processus de transformation du produit traditionnel en produit pharmaceutique classique reste difficile à réaliser dans la mesure où entrent dans sa composition douze extraits actifs de plantes, une modalité qui rend sa mise au point et sa validation par la méthode EBM très complexes. Enfin, une raison pratique peut faciliter l'extension du réseau : l'innovation médicamenteuse pour le traitement du paludisme et du VIH est vivement souhaitée car la biomédecine se trouve encore plus ou moins dans une impasse pour faire face à ces deux pathologies.

\section{Conclusion}

Appliqué à notre objet de recherche, l'objet hybride est un concept heuristique pour décrire et analyser des objets tels que le médicament, objet matériel et social. Les étapes de sa mise au point, de sa validation, de sa distribution et de sa commercialisation s'enchaînent [Garnier, Levy, 2007] et sa «vie » ainsi retracée prend la forme d'un réseau long [Latour, 1991]. L'émergence d'un objet hybride est le résultat d'une action d'hybridation qui suppose l'existence de "réseaux longs ». Elle résulte de la constitution de liens en réseaux de socialité et en particulier de processus de circulation, d'assemblages paradoxaux ou improbables et de nœuds d'interaction entre idées, acteurs, usages ou techniques. La nature hybride d'un objet selon Latour est avant tout associée à l'existence "d'un réseau long » comme condition nécessaire mais en aucun cas suffisante. La chaîne opératoire du médicament est sans aucun doute un "réseau long ». Alors que la tablette Tang est prescrite en traitement combiné dans le programme national $\mathrm{VIH} /$ sida, elle est

22. Par exemple en vente sur le site www.anamed.net 
quasi-inconnue en santé globalisée. D'autres produits de médecine chinoise ou qui en sont issus sont déjà ou susceptibles d'être commercialisés à une échelle mondiale : citons l'artémisinine, l'arsenic trioxide et les extraits actifs de plantes indiquées dans le tableau de $\mathrm{Xu}$ [2011, S.91], testés par des essais cliniques de recherche biomédicale en voie d'obtenir une AMM par des instances reconnues à un niveau international (Agence européenne de médecine, FDA américaine).

Les CTA et la tablette Tang peuvent être considérés comme résultant d'un processus d'innovation en médecine chinoise parce que ces produits ne relèvent pas d'une adaptation de traitements déjà connus. De l'autre côté de la frontière "paradigmatique », pour autant que cette frontière existe encore, c'est-à-dire du point de vue de la biomédecine, il convient aussi de les qualifier de nouveaux produits car des extraits de plantes de la pharmacopée chinoise ont été testés selon les normes de la recherche biomédicale. Néanmoins, en dépit de quelques exemples paradigmatiques, de décennies de recherche et d'investissements financiers importants, les substances actives des compositions de la médecine chinoise traditionnelle restent difficiles à définir, à tester, et leur efficacité compliquée à évaluer et cette médecine est encore considérée comme une médecine complémentaire et alternative dans les pays occidentaux et le reste du monde [Xu, 2011].

Enfin, la distinction « matière médicale historique » et « médicaments botaniques modernes », termes utilisés par les entreprises pharmaceutiques chinoises en recherche et développement et lors de la commercialisation des produits (voir exemple cité de Hu [2008]), tend à s'effacer dans le processus de recherche et de développement des produits, couplé avec le mode de production industrielle et la stratégie de marketing visant à occuper une niche de marché en santé globalisée : un tel processus se retrouve alors au cœur d'enjeux économiques. Par conséquent, des catégorisations locales résultant de la rencontre entre divers savoirs médicaux offrent une perspective plus générale pour (re)définir des nouveaux produits pharmaceutiques industriels, et révèlent en outre voire même avant tout des processus sociaux. 


\section{Bibliographie}

van Agtamael M.A., Eggelte T.A., van Boxtel C.J. [1999], "Artemisinin Drugs in the Treatment of Malaria: From Medicinal Herb to Registered Medication", Trends in Pharmacological Sciences, n²0, p. 199-205.

Appadurai A. (ed.) [1986], The Social Life of Things. Commodities in Cultural Perspective, Cambridge UK, Cambridge University Press, 329 p.

DE BRuyn P.H., Micollier E. [2011], « Diffusion institutionnelle de la médecine chinoise : Typologie des principaux enjeux », Perspectives chinoises, $\mathrm{n}^{\circ} 116$, p. 24-33.

Business/Finance China [2009], “Golden Meditech Successfully completed acquisition of Tang Cao Pian (TCP) business", Press Release to Editors, September 8, 2004.

86

Cambrosio A., Keating P. [2003], « Qu'est-ce que la biomédecine ? Repères socio-historiques », SRMS (Société de la revue médecine/sciences) et EDK Éditions, vol. 19, nº 12, p. 1280-1287.

Coll [2011], “Traditional Asian Medicine”, Nature, vol. 480, n 7378, suppl., p. S82-S115. Duffy P.E., Mutabingwa T.K. [2004], “Drug combinations for malaria: Time to ACT?”, The

Lancet, $\mathrm{n}^{\circ}$ 363, p. 3-4.FISHER R.A., YATES F. [1938], Statistical Tables for Biological, Agricultural and Medical Research, London, Oliver and Boyd, 90 p.

FLECK L. [2005], Genèse et développement d'un fait scientifique, traduction par Jas N. de l'ouvrage de 1935, Paris, Les Belles Lettres, 322 p.

Frank R., Stollberg G. [2004], "Conceptualizing Hybridisation. On the Diffusion of Asian Medical Knowledge to Germany”, International Sociology, vol. 19, nº 1, p. 71-88.

GARnier C., Levy J.J. (dir.) [2007], La Chaîne des médicaments : Perspectives Pluridisciplinaires, Collection Santé et société, CA, Presses Universitaires du Québec, 499 p.

VAn der Geest S., Whyte S.R., Hardon A. [1996], “The Anthropology of Pharmaceuticals. A Biographical Approach", Annual Review of Anthropology, n² 25, p. 153-178.

HiRT H.M., Lindsey K. [2006], « Thé à l'Artemisia annua. Tisane à Artemisia annua une révolution dans l'histoire de la médecine tropicale », Action for Natural Medicine (ANAMED) : http://www.anamed.org/Francais_Home/The_a_1_Artemisia_annua/the_a_1_artemisia_ annua.html (page consultée le 13 avril 2013).

Hobsbawm E., Ranger T. [1983], The Invention of Tradition, Cambridge, Cambridge University Press, 320 p. 
Hoizey D., Hoizey M.J. [1988], Histoire de la médecine chinoise : Des origines à nos jours, Paris, Payot, 293 p.

Hsu E. [2002], "The Medicine from China has 'Rapid Effects': Chinese Medicine Patients in Tanzania", Anthropology and Medicine, vol. 9, n 3, p. 291-313.

Hsu E., [2006], "The History of Qinghao in the Chinese Materia Medica", Transactions of the Royal Society of Tropical Medicine and Hygiene, vol. 100, nº 6, p. 505-508.

Hsu E. [2008], "The History of Chinese Medicine in the People's Republic of China and its Globalization", East Asian Science, Technology and Society: an International Journal, vol. 2, $\mathrm{n}^{\circ}$ 4, p. 465-484.

Hsu E. [2009], “Chinese Propriety Medicines: An 'Alternative Modernity?' The Case of the Anti-Malarial Substance Artemisinin in East Africa", Medical Anthropology: Cross-Cultural Studies in Health and Illness, vol. 28, $\mathrm{n}^{\mathrm{o}} 2$, p. 111-140.

Hsu E. (en consultation avec F. Obringer) [2010], "Qinghao (Herba Artemisiae annuae) in the Chinese Materia Medica", in Hsu E., Harris S. (ed.), Plants, Health, and Healing: on the Interface of Ethnobotany and Medical Anthropology, Oxford, Berghahn Books, p. 83-130.

Hu J. [2008], "From Traditional Chinese Materia Medica to Modern Botanical Drugs", Congress Proceedings, The WHO Congress on Traditional Medicine, Symposium on Medicines. Traditional, Alternative and Complementary, Beijing, Nov. 7-9.

Inda J.X., Rosaldo R. [2002], "Introduction: A World in Motion", in Inda J.X., Rosaldo R. (ed.), The Anthropology of Globalization: A Reader, Massachusetts, Blackwell Publishing, p. 134 .

Klayman D.L. [1985], “Qinghaosu (artemisinine): An Antimalarial Drug from China”, Science, $\mathrm{n}^{\circ} 228$, p. 1049-1055.

87

LAKoff A. [2008], La Raison pharmaceutique, Paris, Les Empêcheurs de Penser en Rond, 319 p.

LABRousse A. [2010], « Nouvelle économie du développement et essais cliniques randomisés : une mise en perspective d'un outil de preuve et de gouvernement », Revue de la régulation, $\mathrm{n}^{\circ} 7$, p. 2-32.

Latour B. [1991], Nous n'avons jamais été modernes. Essai d'anthropologie symétrique, Paris, La Découverte, 206 p.

Li G.Q. [1990], Clinical Trials on Qinghaosu and its Derivatives, Guangzhou, Guangzhou College of Traditional Chinese Medicine, Sanya Tropical Medicine Institute, 90 p. 
Micollier E. [2007A], «Facettes de la recherche médicale et de la gestion du VIH-sida dans le système de santé chinois : un autre exemple d'adaptation locale de la biomédecine », Sciences sociales et santé, vol. 25, $\mathrm{n}^{\circ} 3, \mathrm{p} .31-39$.

Micollier E. [2007B], Neo-Traditional Treatments for AIDS in China: National AIDS Treatment Policy and Local Use of TCM (Traditional Chinese Medicine), abstract 345, session 8.5, AID Simpact International Conference, Marseilles, July 1-4.

Micollier E. [2009A], "Experimenting on Innovative Scientific Versus Traditional Treatments: the Case of AIDS Medical Research in China", in Glymour C., Wang W., Westerstahl D. (ed.), Logic, Methodology and Philosophy of Science: Proceedings of the Thirteenth International Congress, London, University of London, King's College Publications, p. 639-644.

MiCOLlier E. [2009B], « Gestion de l'épidémie et usages de la médecine chinoise. Traitements et recherche dans le contexte de l'extension du traitement médical et social du sida en Chine », in Micollier E. (dir.), « Société chinoise face au sida », Perspectives chinoises, n 106, p. 75-87.

Micollier E. [2010], “At the Margins of Paradigms in Medicines: Treatments and Research in Chronic Diseases in China Today", Maynooth, August 24-27, EASA (European Association of Social Anthropology) Conference, Workshop 072.

MiCOLliER E. [2011], «Un savoir thérapeutique hybride et mobile. Éclairage sur la recherche médicale en médecine chinoise en Chine aujourd'hui », Revue d'anthropologie des connaissances, vol. $5, \mathrm{n}^{\mathrm{o}} 1$, p. 41-70.

OMS [2010], The Pharmacopoeia of the People's Republic of China (PRC).

OMS [2011], Communiqué de presse du 12 janvier, «Il est urgent d'agir pour préserver les traitements antipaludiques: http://www.who.int/mediacentre/news/releases/2011/malaria_ therapies_20110112/fr/index.html (page consultée le 31 janvier 2011).

Ong A., Collier S.J. [2005], Global Assemblages: Technology, politics, and ethics as anthropological problems, Oxford, Blackwell publishing, 494 p.

Sackett D. L., Rosenberg W.M., Gray J.A., Haynes R.B., Richardson W.S. [1996], "Evidence based medicine: What it is and what isn't?", British Journal of Medicine, $\mathrm{n}^{\circ}$ 312, p. 71-72.

ScheID V. [2002], Chinese Medicine in Contemporary China: Plurality and Synthesis, DurhamLondon, Duke University Press, 432 p.

SHAo B.P. [2008], "Clinical effect and safety report on HIV/AIDS patients with Tangcao tablets", Proceedings of the $6^{\text {th }}$ Conference on HIV/AIDS Treatment with TCM, China Asso- ciation of Traditional Chinese Medicine, Anhui, China, December.

TAYlor K. [2005], Chinese Medicine in Early Communist China, 1945-1963: A Medicine of Revolution, London, Routledge, 236 p. 
Tu Y.Y. [2011], “The Discovery of Artemisinin (qinghao su) and Gifts from Chinese Medicine”, Nature Medicine, vol. 17, $\mathrm{n}^{\mathrm{o}}$ 10, p. 1217-1220.

Unschuld P.U. [1986], Medicine in China: A History of Pharmaceutics, Berkeley, CA, University of California Press, $423 \mathrm{p}$.

WANG J., Zou W. [2007], “A General Introduction of HIV/AIDS Treatment with Traditional Chinese Medicine in China", Virologica Sinica, vol. 22, nº, p. 471-475.

Wang J., LiAng B.Y. [2011], "Progress on Research for the Treatment of HIV/AIDS with Traditional Chinese Medecine (TCM) in China", World Journal of AIDS, n 1, p. 104-109.

Whyte S.R., van der Geest S., Hardon A. [2002], The Social Lifes of Medicines, Cambridge, U.K, Cambridge University Press, 200 p.

Woodrow C.J., Haynes R.K., Krishna S. [2005], “Artemisinins”, Postgraduate Medical Journal, $\mathrm{n}^{\mathrm{o}} 81$, p. 71-8.

Wright C.W., Linley P.A., Brun R., Wittlin S., Hsu E. [2010], “Ancient Chinese Methods Are Remarkably Effective for the Preparation of Artemisinin-Rich Extracts of Qing Hao with Potent Antimalarial Activity", Molecules, $\mathrm{n}^{\mathrm{0}} 15, \mathrm{p} .804-812$.

Xu Z. [2011], Modernization: One step at a time, Nature, vol. 489, nº 7378, suppl., p. S90-S92.

Zou W., Liu Y., Wang J., Li H.J., Liao X. [2012], Traditional Chinese Herbal Medicines for Treating HIV Infections and AIDS, Evidence-Based Complementary and Alternative Medi-cine, Review Article, 8p.: http://www.hindawi.com/journals/ecam/2012/950757/ (page consultée le 13 avril 2013).

Sources chinoises

Bencao Gangmu (Traité de Matière Médicale) [1596], Édition moderne, 1975-1996, auteur : LI SHIZHEN, Beijing, Renmin Weisheng Chubanshe, 2 vols., $1526+1634$ p.

Shennong Bencao Jing (Classique de Matière Médicale de Shennong) [1959], auteur : légendaire, $1^{\mathrm{er}}$ siècle, Taipei, Shangwu Yingshuguan, par WEI WUPUSU, 514 p.

Zhou hou bei ji fang (Manuel de Prescriptions des urgences gardées sous la manche) [1983], auteur : Ge HONG (284-363) in Si ku quan shu (Collection des Ouvrages des Quatre Entrepôts), Taipei, Shangwu Yingshuguan, par WEN YUANGE. 